\title{
INVESTIGATION OF THE UNIVERSE BY RADIO ASTRONOMY*
}

\author{
By Prof. A. C. B. LOVELL, O.B.E., F.R.S. \\ Jodrell Bank Experimental Station
}

$\mathrm{A}^{\mathrm{L}}$ LTHOUGH nearly all the major advances in radio astronomy have taken place during the past fifteen years, the basic discoveries were made thirty years ago. At that time Jansky realized that the residual noise in his receiving equipment had a daily sidereal variation and must be the result of radio waves reaching the Earth from outer space, and Appleton in the United Kingdom with Breit and Tuve in the United States, through their studies of the ionosphere, laid the foundation of the radio-echo techniques of radio astronomy. The radio emission from outer space can be received on Earth in the wave-length range from a few millimetres to 10-20 metres. The short-wave end is limited by absorption in the atmosphere and the long-wave end by the ionosphere, and this upper limit tends to vary in sympathy with ionospheric conditions throughout the sunspot cycle. These hindrances will soon be overcome when suitable equipment can be carried in Earth satellites; then it should be possible to determine the true wave-length range of these extra. terrestrial emissions.

Here we are concerned with some of the significant advances made by the application of these techniques to the study of the solar system and the universe, with points of interest from the immediate environment of the Earth to the deepest penetration of the universe.

Some of the earliest successes with the new techniques arose from the study of the transient echoes which are obtained when a radio beam is directed into space. It was shown that these echoes originated from the scattering of the radio waves by the ionized trails of meteoric particles which were evaporating in the height-range of $80-120 \mathrm{~km}$. above the Earth's surface. Methods were developed for the measurement of the velocities of these meteors and later for the determination of the orbits of individual meteor particles. A long-standing controversy was settled when it was shown that the sporadic meteors were members of the solar system and not visitors from interstellar space. New meteor showers active in the day-time sky were discovered and progress has been made in the basic problem of the genesis of this meteoric dust which pervades the solar system.

The extension of these radio echo techniques to the investigation of the Moon and the planets is a more recent activity which required the development of large radio telescopes and powerful transmitters. The lunar work has had two unexpected outcomes which arose from an analysis of the fading of the lunar radio echoes. One has been the discovery that the radio waves are effectively scattered from a rather small portion of the forward hemisphere of the lunar surface; and this led to the demonstration of the possibility of using the Moon as a reflector for round-the-world communication. The other was the proof that a component of the fading arose from Faraday rotation in the ionosphere which has led to * Substance of a Royal Society Tercentenary Lecture, delivered on
July 23 . an important method for measuring the total electron content along the line of sight from the Earth to the Moon. The attainment of planetary echoes is still a difficult technical problem, and so far only limited success has been achieved in the case of Venus. The future of this work is full of promise since it may involve refinement of the value of the solar parallax, the determination of the rotation period of Venus and preliminary information about the nature of the planetary surface and atmosphere.

The emission from the Moon and many of the planets has now been measured in the radio-frequency range, and this has made possible a comparison of the black-body temperatures in the radio and visual regions. The measurements on Jupiter have been of particular interest for two reasons. First, it appears that the effective size of the disk depends on the wave-length, which indicates that the planet may be surrounded by radiation belts. Secondly, the planet exhibits a strong non-thermal and sporadic type of emission on frequencies below $30 \mathrm{Mc} / \mathrm{s}$. The origin of this emission is unknown although it is believed that it may arise from a relatively few places on the planetary surface.

The Sun has been the most exhaustively studied single object in radio astronomy. Various types of emission in the radio-frequency range have now been delineated. The thermal component originates in the solar corona with an extension which depends on the wave-length. In the centimetric range the effective radius is similar to that of the solar disk with pronounced limb brightening. As the wave-length of observation is increased the effective size of the solar disk increases, until at wave-lengths of several metres the coronal radiation can be detected out to many solar radii. Various types of powerful sporadic emission are associated with sunspots and flares, and the mechanism of emission and the relation to the ejection of particles from the Sun remains an active subject of investigation. The recent results obtained by Simpson of Chicago in the American space probe Pioneer $V$ have been of outstanding interest. In this work the cosmic rays from the Galaxy have exhibited a sudden decrease in intensity coincident with solar eruptions, radio emissions and geomagnetic disturbances, when measured at distances of several million kilometres from Earth.

The radio emission from the Milky Way has various origins, the study of which forms a major feature of contemporary research in radio astronomy. The most striking general feature is the resemblance of the intensity isophotes to the concentration of common stars. It has long been realized, however, that the common stars (even if they emit at all) do not contribute significantly to this radiation. The following classes of emission are now recognized:

(a) There are a number of localized, discrete sources of emission (radio stars) concentrated near the galactic plane. A few of these have been clearly associated with galactic nebulosities characterized 
by a filamentary gaseous structure with violent internal motion. These include the three known cases of supernove (the Crab Nebula, and the supcrnovie? observed by Kepler and Tycho Brahe), and the most intense radio source in the sky in Cassiopeia. It seems likely that the Cassiopeia source and the other radio nebulosities may also be supernova remnants tho initial outburst of which has not been recorded.

(b) Thore is a generally distributed emission observable over a wide rango of wave-lengths the isophotes of which are similar to the stellar distribution. At short wave-longths this omission has thermal characteristics and probably originatos in froe-free transitions from the movoment of olectrons in the field of the interstellar protons. At long wavelengths the intensity and spectrum of the radiation are incompatible with such an origin, and this emission is belioved to be synchrotron radiation arising from the relativistic motion of the electrons in the galactic magnetic ficld.

(c) Radio emission is observable from regions far beyond the galactic disk of visible stars. This halo or corona of emission is thought to originate in the same way as the long-wave radiation in the Galaxy, that is, from the relativistic motion of electrons in the galactic magnetio field.

(d) In 1951 observers in Leyden, Harvard and Sydney almost simultaneously established the existence of line emission from the neutral hydrogen clouds in the Galaxy on a frequency of $1420 \mathrm{Mc} / \mathrm{s}$. This emission is associated with the transition between two levels in the hyperfine structure of the ground-state of the hydrogen atom. The two levels correspond with the two possible orientations of the electron spin in the magnetic fiold of the proton. The original suggestion was made in 1945 by van de Hulst, and since the practical demonstration of the existence of this line remarkable progress has been made, particularly by the Leyden school, in observations of the Doppler frequency shift and the derivation of the motion of the galactic hydrogen clouds. This work has given the first detailed insight into the spiral structure of the Galaxy, and is now in process of extension to determine the hydrogen content of extragalactic nebulx.

An important outcome of the existence of the observable hydrogen lino emission is the possibility of measuring the galactic magnetic field by observing the Zeeman effect. The magnetic field should split the line into three components with an extreme frequency difference of $2.8 \mathrm{Mc}$./s. per gauss. Since the interstellar field is not expected to be more than $10^{-5}$ gauss the maximum observable shift is likely to be of the order of 30 c.p.s. Experiments are in progress on this problem at Jodrell Bank, and so far an upper limit of $4 \times 10^{-6}$ gauss has been set for the interstellar magnetic field.

Even a cursory inspection of the intensity plot of a high-resolution radio survey of the slky shows that there are prominent features which are not explicable in terms of the emission from the Milky Way. The most striking fenture is the existence of large numbers of localized sources (radio stars) which seem to have a nearly isotropic distribution. A small number of these (two or three dozen) can be associated with the noarer normal types of extragalactic nebulx. The Andromeda nebula ( $M$ 31) has been studicd in some detail, and as a radio emitter it is found to be essentially similar to the Milky Way, including tho existence of an extensive corona around the Galaxy. Studies of the other spiral nebulæ within the range of presentday radio telescopes are still in progress, but the existing evidence is that they also conform to this general pattern.

The problem of the majority of these isotropically distributed sources which are not associatied with normal extragalactic nebula is believed to be one of the most significant issues in astronomy to-day. It is thought possible that the failure to associate these with visible objects may be because they lie at very great distances in the cosmos. The direct evidence for this arises from a few cases where colliding or interacting nobulæ at great distances have been associated with rather strong radio sources. The most outstanding case is that of the galactic collision in Cygnus at a distance of 700 million light years which is related to the second strongest radio source in the sky. The disparity between the visual and radio luminosity of this source is such that a similar event at ten times the distance could only be observed optically with great difficulty but would still be easily recorded as a radio source. Recently one of these radio sources, the apparent angular diameter of which is about 8 sec. of arc, has been related to a rich cluster of nebule at a distance of 6,000 million light years with a recessional velocity amounting to 46 per cent of the velocity of light. The lecture concluded with a reference to the cosmological issues involved in this work, and to the experiments now in progress which are believed to be giving information about the condition of the cosmos at distances of many thousands of millions of light years.

\title{
A NEW THEORY OF THE ANGIOSPERM FLOWER
}

\author{
By DR. R. MELVILLE \\ Royal Botanic Gardens, Kew
}

\begin{abstract}
$\mathrm{T}$ HE interpretation of the Angiosperm flower as a structure built up from modified leaves bearing either ovules or microsporangia dates back to Goethe's essay on the metamorphosis of plants'. Most attention and much controversy has been focused on tho structure of the ovary, which in its simplest state was supposed to have been produced by tho infolding of a loaf bearing ovules on its margin to form a boxlike structure similar to the pod of a pea. Tho carpel
\end{abstract}

theory does account for many of the facts of floral structure; but numerous incongruities are glossed over or avoided by its proponents. Alternative theories havo been put forward from time to time, one of the more recent being Suunders's theory of carpol polymorphism². 'This was shown to be untcnable by Eames, in a paper in which he expounded the modorn form of the carpel theory, that assumes the primitive carpel to have been a palmate leaf 